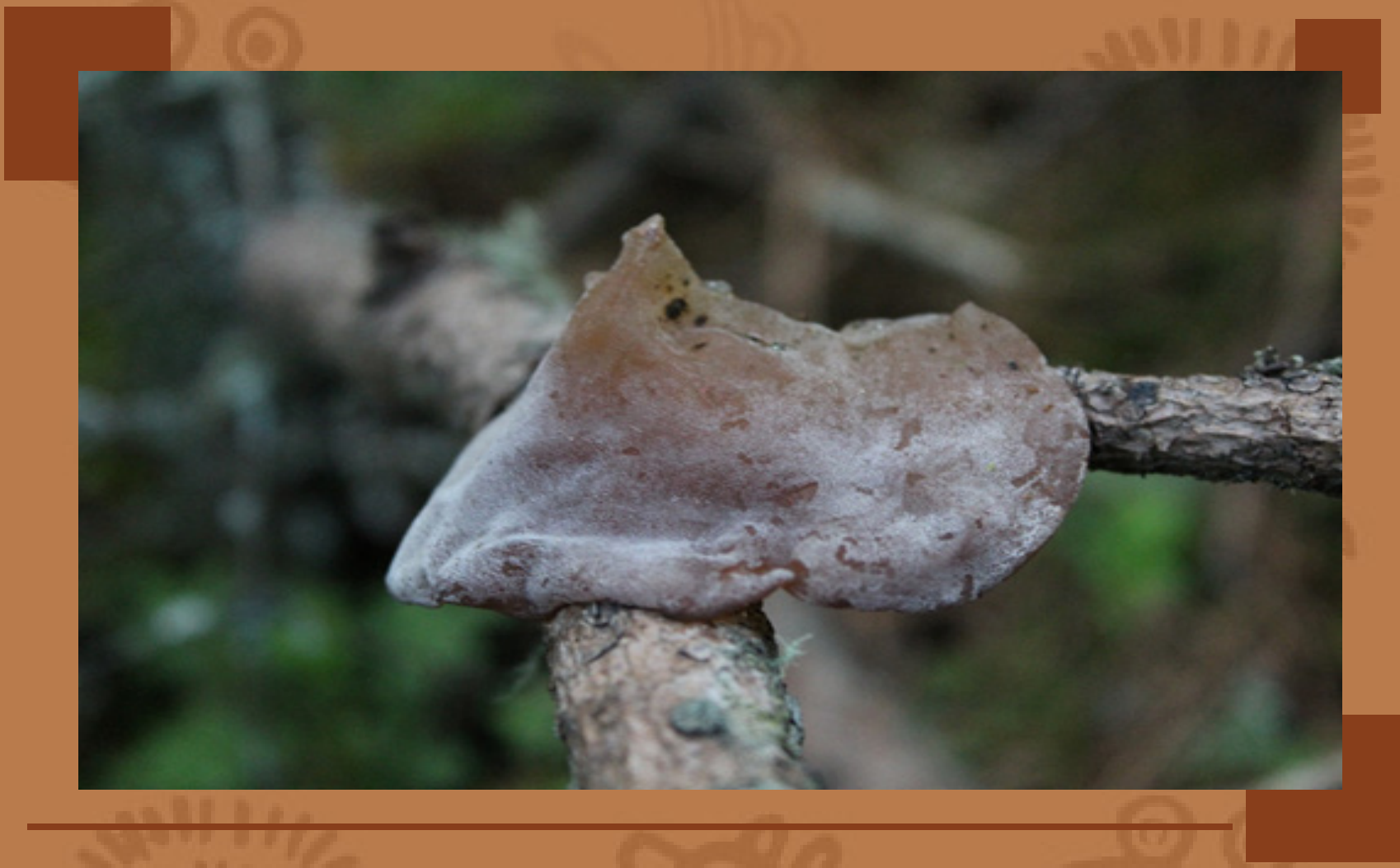

Experiencia educativa@en Etnomicología con adolescentes de San Pedro Tlalcuapan (México)

Estudios Interdisciplinarios 


\section{Experiencia educativa en Etnomicología con adolescentes de San Pedro Tlalcuapan (México)}

\section{Educational experience in Ethnomycology with adolescents from San Pedro Tlalcuapan (Mexico)}

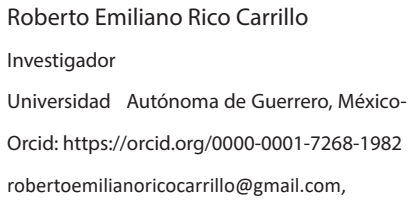

Roberto Emiliano Rico Carrillo robertoemilianoricocarrillo@gmail.com,

\author{
Adriana Montoya Ezquivel \\ Investigadora de la Universidad Autónoma de \\ Tlaxcala, México \\ Orcid: https://orcid.org/0000-0002-6531-3521 \\ ametnomicol@hotmail.com
}

\author{
Juan Camilo Cardona Castaño \\ Investigador \\ Universidad Santo Tomas, Colombia \\ Orcid:https://orcid.org/0000-0002-9631-9870 \\ juancamilocardonacastano@gmail.com
}

Recibido: 21-05-2021

Aceptado: 03-06-2021

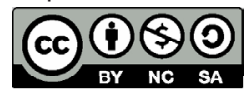

Copyright $\odot 2021$ UNAN-Managu
Todos los Derechos Reservados.

\section{Resumen}

La investigación documentó la experiencia educativa ambiental con jóvenes, la interacción con el medio natural y reino de los hongos. La educación ambiental contribuyó al reconocimiento del espacio, la vida y el diálogo con la naturaleza, principios ligados a la presente investigación. Percepciones y conocimientos en adolescentes sobre la naturaleza de comunidades rurales de San Pedro Tlalcuapan, son reflejo identitario al nivel social y cultural. El estudio se ubicó en el paradigma cualitativo de tipo narrativo, se desarrolló talleres de educación ambiental en grupo focal de 26 estudiantes de secundaria respondiendo a preguntas detonantes sobre el ambiente, la ecología de hongos silvestres; usos y saberes. Por medio del diario de campo, se analizó la información en tres constructos: usos y conocimientos de los hongos silvestres y la importancia de dichos organismos en la vida diaria. La investigación arrojó, preparaciones gastronómicas de hongos en la vida rural, el grupo identificó que los hongos son activo natural, estigmatizado por comunidades "civilizadas", asimismo, son herencia ancestral. Se concluyó, el grupo focal confirió respuestas sobre los saberes etnomicológicos y la relación que tienen dichos recursos en comunidades rurales relativamente aislada de urbes y concesiones antropocéntricas, es la educación ambiental la que develó el entramado de interrelación: comunidad y la conservación de costumbres.

Palabras claves: experiencia educativa, etnomicología, saberes ambientales, hongos silvestres, comunidad rural

\section{Abstract}

The research documented the environmental educational experience with young people, the interaction with the natural environment and the kingdom of fungi. Environmental education contributed to the recognition of space, life and dialogue with nature, principles linked to this research. Perceptions and knowledge in adolescents about the nature of rural communities of San Pedro Tlalcuapan, are a reflection of identity at the social and cultural level. The study was located in the qualitative narrative paradigm, environmental education workshops were developed in a focus group of 26 high school students responding to triggering questions about the environment, the ecology of wild mushrooms; uses and knowledge. Through the field diary, the information was analyzed in three constructs: uses and knowledge of wild fungi and the importance of these organisms in daily life. The research showed, gastronomic preparations of mushrooms in rural life, the group identified that mushrooms are a natural asset, stigmatized by "civilized" communities, also, they are ancestral inheritance. It was concluded, the focus group gave answers about ethnomicological knowledge and the relationship that these resources have in rural communities relatively isolated from cities and anthropocentric concessions, it is environmental education that revealed the interrelation network: community and the conservation of customs.

Keywords: educational experience, ethnomycology, environmental knowledge, wild mushrooms, rural community 


\section{Introducción}

Los hongos fundamentales dentro de los ecosistemas, descomponedores por excelencia y contribuyen a la degradación de materia orgánica, son diferentes de las plantas o el reino vegetal, puesto que no hacen fotosíntesis y su pared externa está provista de quitina: proteína estructural del hongo y otras características morfológicas y fisiológicas (Ruan, Ordaz, 2015).

El desconocimiento de los hongos silvestres: usos, conocimientos, aspectos biológicos; servicios ecosistémicos y el significado ancestral en comunidades rurales e indígenas, es evidente y persiste en la actualidad, Montoya et al. (2019). La investigación documenta las experiencias durante la implementación de un programa educativo en la comunidad de San Pedro de Tlalcuapan donde 26 adolescentes participaron de dicha investigación.

Los adolescentes de las comunidades rurales en el municipio de San Pedro TlalcuapanTlaxcala, adoptan los hongos silvestres brindados por el territorio que vive, dando diversos usos en la vida diaria, por ejemplo: incorporación en la dieta, cultivos de forma agroecológica, valor económico y con gran significado de identidad cultural, Ureta (2020). Sin embargo, la documentación sobre usos, aprovechamiento y conservación de hongos silvestres en el territorio de San Pedro, presenta falencias, lo anterior se debe porque pocos proyectos de intervención logran ahondar en los saberes ambientales, documentando experiencias significativas sobre recursos naturales que son importantes en las comunidades rurales. Por tanto, la investigación desarrolló una recopilación de significados y experiencias desde la educación ambiental con jóvenes (Lara, Romero, Burrola, 2013).

Asimismo, San Pedro Tlalcuapan, crucial para el desarrollo del programa educativo, teniendo como escenario la biblioteca del municipio, contexto que sirvió en la interacción con el grupo focal y posterior, se realizaron sesiones educativas acompañadas de salidas de campo fortaleciendo el dinamismo de las actividades y la construcción de conceptos a través de la interacción con el medio natural por parte de los participantes.

Las comunidades logran interacción con el ambiente, fundamental para la comprensión de costumbres, saberes y diálogos que se construyen desde el entorno, esto permitió que los participantes fuesen abiertos, atentos y con gran disposición de dialogar sin temores sobre usos, conocimientos y experiencias que la naturaleza les brinda a través del reino fungi (Ramírez, Montoya, Caballero, 2014).

\section{La educación ambiental}

De acuerdo con, Cardona, Lamprea, Cubides (2021), la complejidad de la educación ambiental resultó ser relevante en la investigación para abordar problemas ambientales, consistió en ser una dimensión clave para la creación de constructos asociados a la interacción que los jóvenes hacen de su vida diaria con el mundo de los hongos. De acuerdo con, Nieto, (2018), se entiende como educación ambiental: aquellas estrategias que se desarrollan en el espacio con el propósito de abarcar un fenómeno ambiental a través de elementos didácticos, lúdicos; observacionales y experienciales, con discurso pedagógico que contribuye a la compresión y aprendizaje de problemas del entorno. 
Con base en lo anterior, poca existe documentación sobre las percepciones del mundo de los hongos en los jóvenes de San Pedro. Por ello, la educación otorga un abordaje complejo para construir un conocimiento participativo Aparicio, Ostos, (2021). De tal modo, de la investigación surgieron: construcción de diálogos de saberes, donde los hongos del territorio de San Pedro Tlalcuapan son el punto de reflexión (Rosetti, 2019).

\section{El dialogo de saberes}

Los diálogos de saberes son aquellas narrativas, interacciones, símbolos; creencias y formas en que las comunidades logran comunicar un entramado identitario y cultural Piñeros, (2021). Es así, como el conocimiento, usos y percepciones del medio natural que expresó el grupo focal, brindó a la investigación todo un acervo propio de la cultura de los participantes, inmersa en la construcción territorial Cabrera, Jiménez, Pérez (2019). Lo anterior, habilitó la comprensión y documentación para la investigación a través del dialogo con adolescentes sobre la importancia del reino fungi (hongos), en su vida diaria. Dicha compresión logró anteponerse a cualquier juicio académico, ético y/o moral y formó parte sustancial dentro del programa educativo.

\section{La interacción con el medio natural}

El hombre y la naturaleza interactúan a través del uso y aprovechamiento de los recursos naturales. Cuya, interacción es fundamental, porque permitió al hombre a lo largo de la historia un estado de bienestar Ruan, (2018). El medio natural, no se desliga de las necesidades humanas, porque existe un alto nivel de dependencia del hombre sobre la naturaleza. Dicha dependencia, cobró valor simbólico en cuanto el hombre reconoció que el medio natural no solo le sirve como factor económico, también significa la vida, la identidad y lo cósmico Jiménez, Thomé, Borrula, (2016). De tal modo, emerge una interacción de tipo simbólica, no lejos sucedió dentro de la presente investigación, el grupo focal reconoció todo un sistema de valores y oportunidades que la naturaleza les brinda a través de su propio territorio, con abundantes hongos, siendo sustento económico, cultural e identidad que no se puede fragmentarse dentro de la interacción con el medio natural (Servín, Alarcón, 2018).

\section{Metodología}

El manuscrito es el resultado de investigación desarrollada en San Pedro Tlalcuapan, en el marco del proyecto "Introducción al reino de los hongos", proyecto dirigido a jóvenes del sector rural de dicho municipio.

La investigación es cualitativa de tipo narrativa; la narración es un relato que se da en el contexto investigativo, incluye, lenguaje visual, sonoro, símbolos, texto y verbo, elementos que son analizados en cualquier investigación de es este tipo de naturaleza. Jiménez, Comet, (2016). La narrativa como diseño, permitió dentro de la presente investigación abordar discursivamente el problema sobre carencia de datos experienciales de educación en el contexto de etnomicología en adolescentes. 
La investigación desarrolló 5 talleres de educación ambiental, los temas tratados; usos y conocimientos del reino del hongo. Previo a los encuentros se aplicó una evaluación diagnostica para saber los conocimientos previos del grupo de participantes. Fueron 26 participantes entre las edades de 13 a 17 años, de secundaria que asistían a la biblioteca del municipio de San Pedro Tlalcuapan, los participantes voluntariamente decidieron participar.

\section{Recolección de la información}

Se realizaron preguntas detonantes cuyas respuestas fueron consignadas en el diario de campo: percepciones, consideraciones; opiniones y gestos, etc.

\section{Análisis de la información}

Con base en la revisión teórica de la investigación, se analizó de forma a priori las siguientes dimensiones: usos de hongos, conocimientos de hongos e interacción con los hongos silvestres, logrando análisis por medio constructos argumentales, nutrido con la información recogida en campo a través de la observación directa, el dialogo directo con los participantes y las notas de diario de campo.

\section{RESULTADOS}

\section{La importancia de los hongos en la vida diaria.}

Los jóvenes comprendieron la complejidad, la importancia y la relación de los hongos silvestres con su vida Alonso, Et. Al. (2014). Dicha comprensión es de forma simbólica, porque la naturaleza ofrece bienestar y en si misma presenta valor cósmico, de vida e identidad entre los participantes, es decir: ellos guardan un equilibrio con el medio natural, evidenciado la importancia sobre el recurso natural, más aún se fortalece dicha interacción, reconociendo que el bosque es una fuente de valor inconmensurable que provee un continuo bienestar; conseguir hongos para su alimentación es un ritual, donde adultos mayores trasmiten el conocimiento a sus menores, sobre aquellas especies que se deben ingerir dentro la dieta. Los jóvenes complejizaron las respuestas, reconociendo la importancia ambiental que los hongos tienen dentro de su vida y territorio e incluso construyeron la relación con el medio natural, hacia el respeto por la naturaleza y el agradecimiento por la misma en brindar un alimento tan importante y con tantas propiedades nutricionales.

Asimismo, los participantes se relacionan con el reino fungi, desde un sistema espiritual, es decir: respetan a estos organismos, saben que constituyen una fuente económica y de valor social y ven en dichos recursos subsistencia de todo tipo, por ejemplo: su alimentación no sería la misma y reconocen que el territorio de San Pedro Tlalcuapan tiene vastos conocimientos sobre usos sustentables de los hongos. Se evidenció un nexo de los participantes con el sistema natural de respeto y sostenibilidad de los recursos naturales.

Dentro de la investigación se realizaron algunas preguntas detonantes de las cuales surgieron los siguientes hallazgos. ¿Cuál es la importancia de los hongos en el medio silvestre? 
El participante 1:

"Lo que mis padres me enseñaron es que lo hongos descomponen materia orgánica, fundamental para otras especies"

La respuesta del participante 1, demuestra que los hongos se encuentran en profunda relación con el suelo y otros organismos vivos, bacterias y plantas.

El participante 5:

"Los hongos no son plantas....... ellos, tienen que fabricar su propio alimento, ;ay!... como es que se llaman, son heterótrofos"

La respuesta del participante 5, es una de las características fundamentales que diferencia los reinos vegetal y fungi, se demostró con dicho argumento que los organismos heterótrofos logran tener el alimento a través de la descomposición, por tanto, son saprofitos.

Se evidenció, conocimientos previos sobre los hongos silvestres y la importancia al nivel ecológico, lo anterior puede explicarse que las comunidades entre más dependan de un recurso natural, implica más conocimiento del mismo.

\section{Participante 17:}

"Por ejemplo, el hongo amarillo algunos visitantes chilangos creen que por su color es tóxico, es comestible y lo encontramos donde existe mucha hojarasca mojada".

El participante 17, se refiere al hongo con el nombre común "Amarillo" Amanita basii, su respuesta tiene tres consideraciones: no se puede pensar que todo color encendido en los hongos indica toxicidad, segundo la hojarasca está indicado la asociación vegetal y el sustrato que descompone para otros organismos y tercero, la idiosincrasia de algunas personas que no pertenecen a la comunidad, con la palabra "Chilango" esto hace referencia aquellas personas que son citadinas de la ciudad de México, lo cual deja al descubierto un entramado de valores entre y constructos culturales de las personas de las grandes urbes.

\section{Conocimientos hongos}

El conocimiento de hongos silvestres, sigue restringido en algunas comunidades, en la actualidad pocos asentamientos rurales y urbanos aprovechan los recursos micológicos que la naturaleza brinda y el territorio provee. Actualmente en comunidades rurales de Tlaxcala aprovechan los hongos de forma alimentaria, bien sabe, los hongos macroscópicos tienen un gran valor proteico indispensable en la salud nutricional de las comunidades rurales que los consumen.

Los participantes, consideraron: que los hongos son importantes dentro del ecosistema, porque descomponen materia orgánica, esencial para generar nutrientes en el suelo y ser aprovechados por otros organismos, como: plantas, pedofauna, etc. 


\section{Raíces}

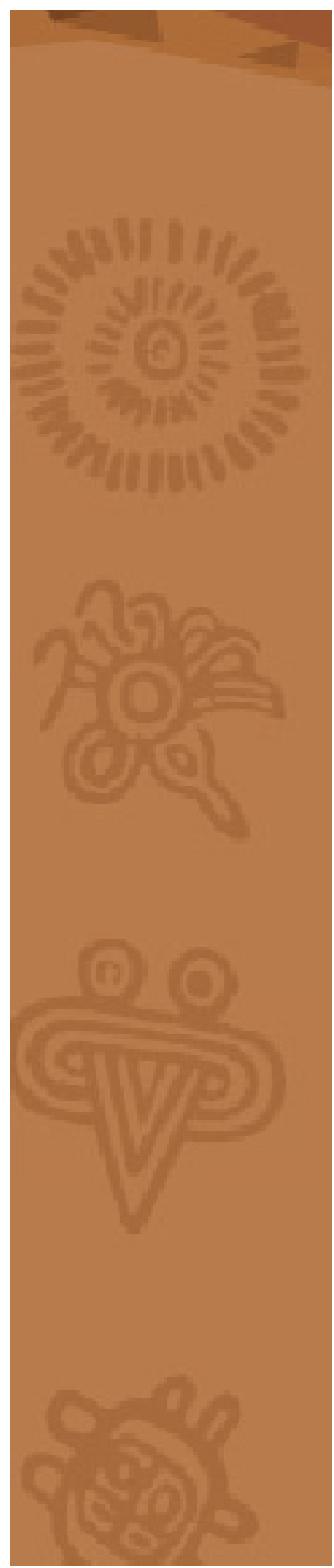

De lo anterior, se identifica que el grupo de participantes, entienden y comprenden la dimensión e importancia ambiental del reino fungi dentro del equilibrio ecosistémicos, demostrando que los participantes identifican el valor ecológico de dichos organismos.

Asimismo, dentro de las preguntas detonantes se les formuló la siguiente: ¿Porque es importante conocer sobre los hongos silvestres?.

\section{Participante 19:}

"El conocimiento de los hongos silvestres nos ayuda... A saber, cuáles se pueden comer y cuáles no, donde encontrarlos y porque cuidar la zona donde los encontramos"

Con la anterior respuesta del participante se analizaron varios aspectos: primero, reconocen en los hongos silvestres un valor alimentario, posterior el conocimiento de dichos organismos implica en sí mismo la comprensión del territorio, dado que saben dónde los pueden encontrar.

Con base en lo anterior, los jóvenes conocen que la especie Amanita muscaria es altamente tóxica.

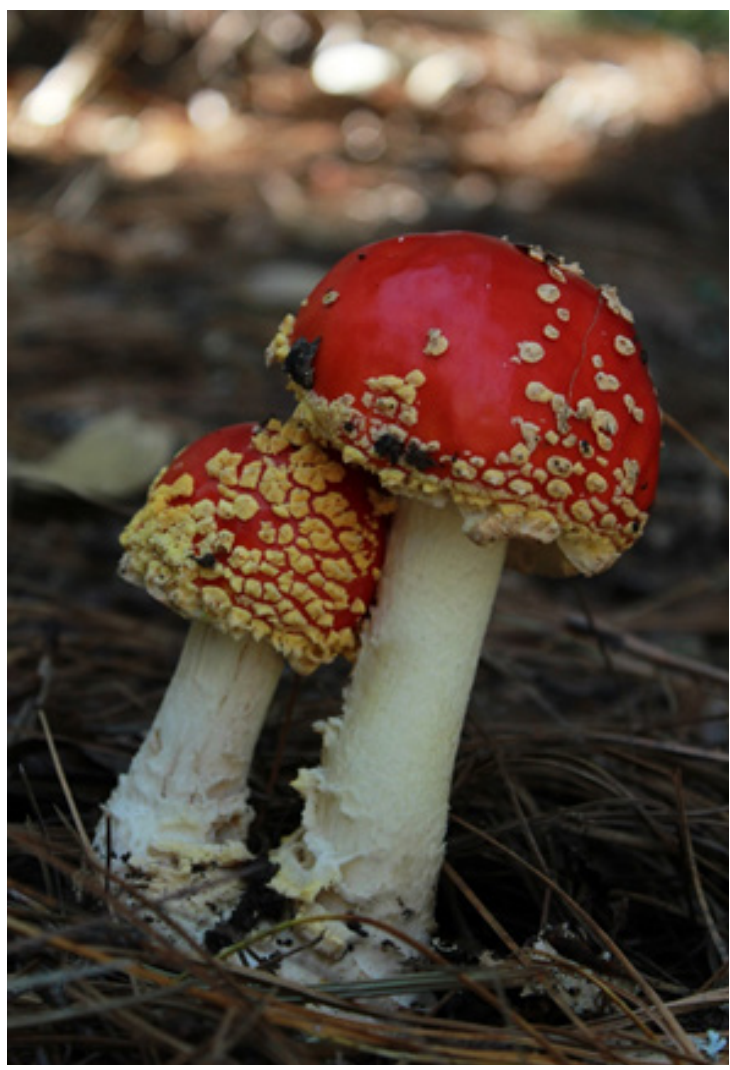

Especie Amanita Muscaria Foto: Fuente propia 
Participante 21:

"Yo se las partes de los hongos, ellos tienen piecito, sombrero, libro, patita y no tienen flores se reproducen por un polvillo que es transportado por el viento".

Otros de los hallazgos importantes dentro de la investigación, los participantes conocen las partes de los hongos y las identifican con un lenguaje coloquial, además, saben sobre las características reproductivas de los hongos, denominando "Polvillo" equivalente en el lenguaje científico a las esporas, además saben que el viento transporta las esporas, a este fenómeno se le conoce científicamente con el nombre de "anemócoria". El anterior concepto según Méndez, López, (2016), es la dispersión de propágulos que logra viajar con el viento, para posterior germinación.

"piecito", estípite; “sombrero"; píleo, "libro"; láminas y "anillo” es velo.

\section{Un territorio para los hongos y los adolescentes}

El territorio de San Pedro Tlalcuapan, es un universo en términos micológicos, durante las salidas de campo los participantes no solo desarrollan una filiación por los hongos, también este conocimiento se traduce en una interacción continua de respeto por la naturaleza y el espacio. El reconocimiento del territorio y pervivencia de los participantes, permitió a lo largo del tiempo la compresión y la complejidad de su espacio y bienestar, cohabitado por un sistema natural donde la clave ha sido promover un lazo de convivencia y conservación constante con el reino fungí. Sin duda, al ser un territorio de coexistencia entre hongos y comunidades rurales favorece la conservación y el aprovechamiento de los recursos naturales: bosque, suelo, hongos, aguas y la interacción constante que dichos recursos tiene con la comunidad, facilitando el aprendizaje en adolescentes de tal forma, que logran retroalimentar el conocimiento sobre los hongos y el municipio de San Pedro Tlalcuapan (Vargas, Zúñiga, 2021).

Participante 14:

"Para mi el territorio es fundamental porque es nuestra dispensa alimentaria, sin suelos fértiles no tendríamos hongos y sin hongos no tendríamos alimento".

Con base en la anterior respuesta, el participante hace un reconocimiento del territorio al nivel de subsistencia, y trae a colación un concepto que se esbozará más adelante, "la seguridad alimentaria".

Dentro de lo usos que la comunidad da a la especie Amanita basii, se encuentra: caldos, tamales y quesadillas. 


\section{Raíces}

Revista Nicaragüense de Antropología Año 5 No.9| 2021 Enero - Junio
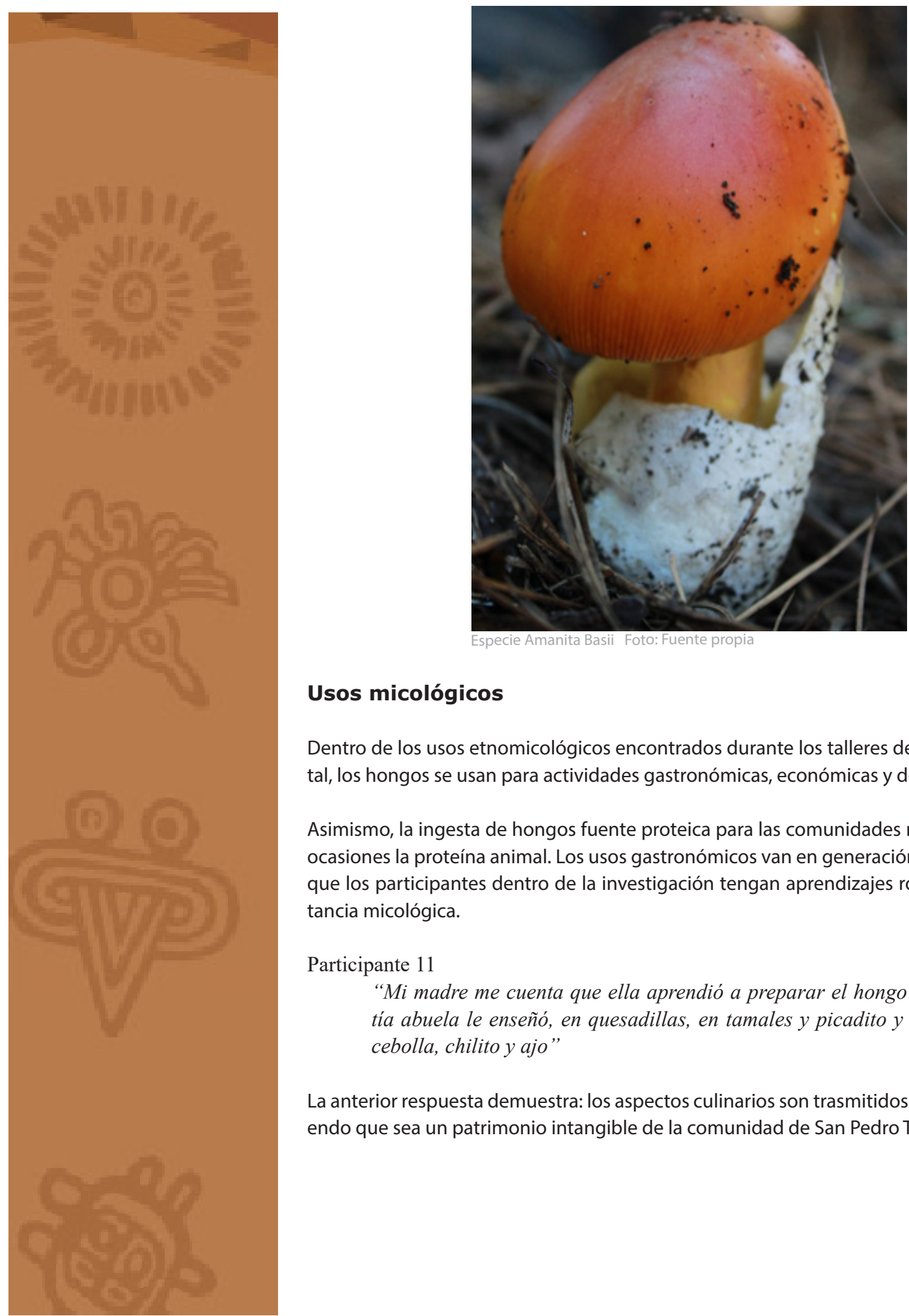

Especie Amanita Basii Foto: Fuente propia

\section{Usos micológicos}

Dentro de los usos etnomicológicos encontrados durante los talleres de educación ambiental, los hongos se usan para actividades gastronómicas, económicas y de conservación.

Asimismo, la ingesta de hongos fuente proteica para las comunidades rurales, supliendo en ocasiones la proteína animal. Los usos gastronómicos van en generación lo que ha facilitado que los participantes dentro de la investigación tengan aprendizajes robustos de la importancia micológica.

\section{Participante 11}

"Mi madre me cuenta que ella aprendió a preparar el hongo amarillo porque mi tía abuela le enseñó, en quesadillas, en tamales y picadito y salteado de ojo con cebolla, chilito y ajo"

La anterior respuesta demuestra: los aspectos culinarios son trasmitidos de generación, haciendo que sea un patrimonio intangible de la comunidad de San Pedro Tlalcuapan. 


\section{La agroecología comunitaria de los hongos}

El factor agroecológico, durante el dialogo con los jóvenes, dentro de los talleres de educación ambiental develó, dicha categoría de tipo emergente. Si bien, la agroecología se fusiona con la gastronomía sin barrera generacional, dicha forma de interactuar con el suelo, también ha hecho que las comunidades rurales y los participantes sepan o tengan principios básicos del cultivo de hongos, esto ha generado una sustentabilidad alimentaria en el municipio de San Pedro Tlalcuapan.

Las comunidades del área de estudio, conservan el bosque sin alterar dicho ecosistema con acciones antropogénicas. El bosque para ellos es un huerto, la comunidad conserva el suelo y promueve la relación sustentable con el ecosistema, tratan de no intervenir el monte para evitar altos niveles de luz solar, permitiendo que la humedad no escape y proliferen con mayor facilidad los hongos, sacan estrictamente lo necesario del ecosistema y la comunidad no usa agroquímicos que afectan pedofauna.

Participante 13.

"Para la mejor forma para nosotros de obtener los hongos o de cultivarlos es conservando el bosque”

Con base en la anterior, respuesta la seguridad alimentaria depende del bosque tanto participantes como comunidad rural entienden la importancia de dicha sustentabilidad. La respuesta del participante habla claro del equilibrio que la naturaleza tiene con la comunidad, es más importante conservar que explotar.

El hongo oreja de ratón Auricularia auricula, es usado por comunidades rural al nivel medicinal para el dolor, y tiene usos gastronómicos.

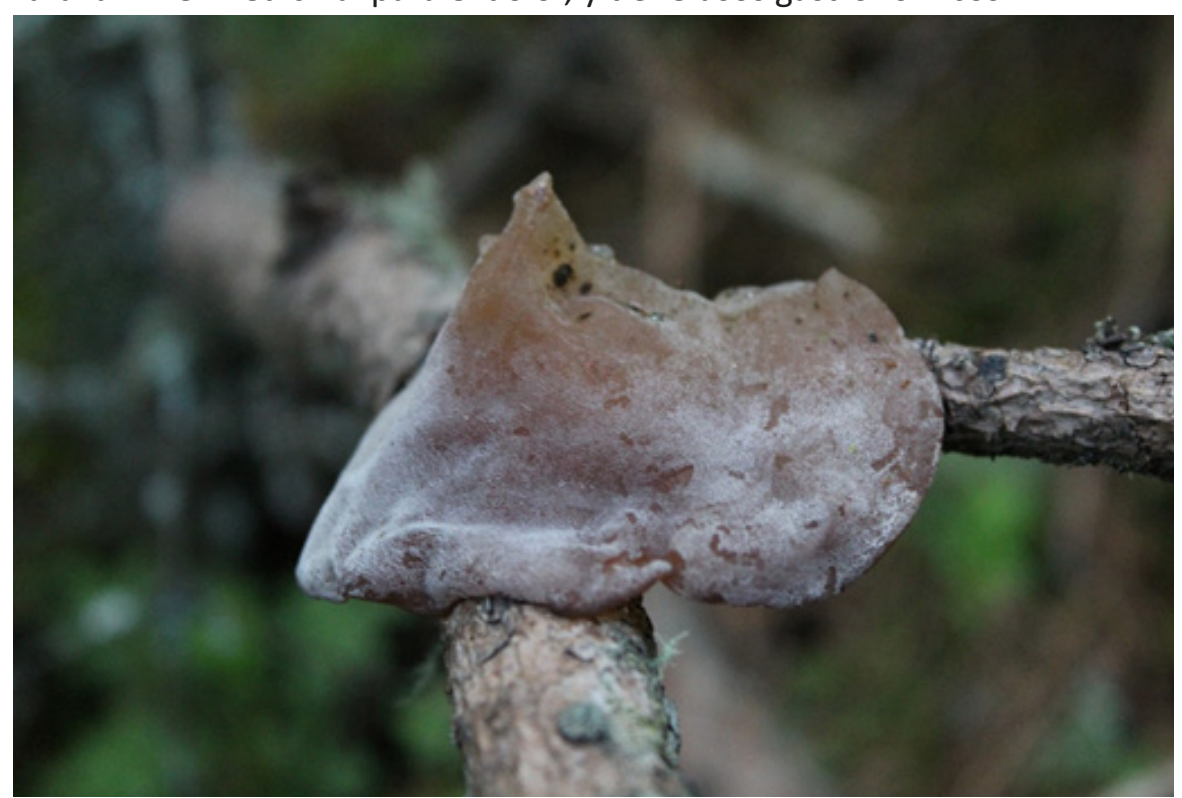

Hongo Oreja de ratón, Auricularia Auricula Foto: Fuente propia 


\section{CONCLUSIONES}

Existe una gran relación de la comunidad de San Pedro Tlalcuapan con el uso, aprovechamiento y la interacción de los hongos. Dentro de la investigación se evidenció que hay traspasó de conocimiento generacional, se evidencia forma implícita dentro del discurso de los participantes, las salidas campo y el conocimiento del bosque introducción involuntaria de la sustentabilidad ambiental lo cual es favorable para conservar cualquier recurso natural.

Usos de los hongos por comunidades rurales

\begin{tabular}{lll}
\hline \multicolumn{1}{c}{ Nombre común } & \multicolumn{1}{c}{ Nombre científico } & \multicolumn{1}{c}{ Usos } \\
\hline Amarillo o de huevo & $\begin{array}{l}\text { Amanita basii Guzmán \& Ram. Guill } \\
\text { Champiñon }\end{array}$ & Gastronómicos \\
& Hypomyces lactifluorum (Schwein.) Tul. \& C. Tul & Gastronómicos \\
Chilnanzi naranja & Morchella snyderi M. Kuo \& Methven & Gastronómicos \\
Chipotle & Turbinellus floccosus (Schwein.) Singer & Gastronómicos \\
Corneta & Lactarius deliciosus (L.) Gray & económicos \\
Enchilado & Ramaria rubricarnata Marr \& D.E. Stuntz & económicos \\
Escobeta amarilla & Ramaria rubripermanens Marr \& D.E. Stuntz & económicos \\
Escobeta rosa & Pleurotus opuntiae & económicos \\
Hongo de maguey & Amanita muscaria & cósmico \\
Hongo rojo malo & Auricularia auricula-judae Bull. & Tóxico- cósmicos \\
Oreja de ratón & Suillus pseudobrevipes A.H. Sm. \& Thier & salud \\
Pancita & Russula xerampelina (Schaeff.) Fr. & Gastronómicos \\
Pastelito & Helvella crispa Bull. & Gastronómicos \\
Soldado & Lactarius indigo (Schwein.) Fr & Gastronómicos \\
Tecax azul & Lyophyllum aff. decastes (Fr.) Singer & Gastronómicos \\
Tsenzo & Hebeloma aff. mesophaeum (Pers.) Quél. & Gastronómicos \\
Xolete & & Gastronómicos \\
\hline
\end{tabular}

Nota: construcción propia. (2018), la identificación de hizo en campo durante las salidas de campo, dicha construida con la información de los participantes, a través de nombres comunes ayudaron a la identificación de especies.

Los hongos Morchella snyderi; Hypomyces lactifluorum; Suillus pseudobrevipes; Auricularia auricula tienen usos gastronómicos, dichos organismos están incorporado en la dieta de las comunidades rurales, haciendo parte de la oralidad de dicha comunidad.

Los participantes reconocen las partes de los hongos, dicha apropiación del conocimiento se lo han logrado por las generaciones antecesoras, permitiendo la conservación del dialogo de saberes. El territorio es un beneficio que ellos tienen, cuentan con altos niveles de biodiversidad micológica.

Es necesario seguir documentando a través de la educación ambiental y el dialogo de saberes formas de entender la construcción territorial, la sustentabilidad y la interacción las comunidades con los servicios ambientales. 


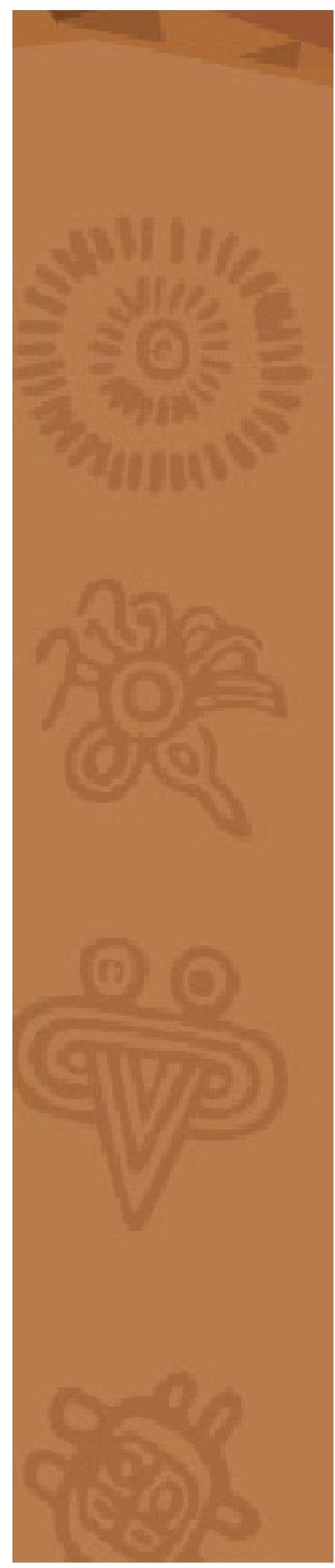

\section{Bibliografia}

Alonso, A, L. Montoya, A. Kong, A. Estrada, T. \& Garibay, O. (2014). The cultural significance of wild mushrooms in San Mateo Huexoyucan, Tlaxcala, Mexico. Jour. Ethnob. Ethnomed. 10 (27)

Aparicio, G. O. \& Ostos, O. O. (2021). Pedagogías emergentes en ambientes virtuales de aprendizaje. Revista Internacional De Pedagogía E Innovación Educativa, 1(1), 11-36. https://doi.org/10.51660/ripie.v1i1.25

Cabrera, S. L. Jiménez, M. F. Pérez, S. J. (2019). Etnobiología De La Comunidad Indígena Salinas De Nahualapa, Tola (Rivas, 2019) Raíces: Revista Nicaragüense de Antropología. 4 (8), 22-40. DOI: https://doi.org/10.5377/raices.v4i8.10629

Cardona, C. J. Lamprea, Z. M. \& Cubides, S. F. (2021). Sobre el concepto de cambio climático e implicaciones: Construcción desde el aula. Revista Internacional De Pedagogía E Innovación Educativa, 1(2), 87-102. https://doi.org/10.51660/ripie.v1i2.39

Jiménez, C. E. \& Comet, W. C. (2016). Los estudios de casos como enfoque metodológico. ACADEMO Revista de Investigación en Ciencias Sociales y Humanidades, 3 (2) Recuperado: https://revistacientifica.uamericana.edu.py/index.php/academo/article/ view/54/52

Jiménez, R, E. Thomé, O. H. Burrola, A. C. (2016). Patrimonio biocultural, turismo micológico y etnoconocimiento. El Periplo Sustentable, 30 (1), 180-205. En: http://www. redalyc.org/articulo.oa? $\mathrm{id}=193443689007$

Lara, V. F. Romero, C. A. Burrola, A. C. (2013). conocimiento tradicional sobre los hongos silvestres en la comunidad Otomí de San Pedro Arriba; Temoaya, Estado de México. Agricultura, Sociedad y Desarrollo, 10 (3), 305-333. https://www.redalyc.org/ articulo.oa? $\mathrm{id}=360533096003$

Méndez, B \& López, H. (2015). Síndromes de dispersión de diásporas de las especies arbustivo y arbóreas de tres tipos de coberturas del Parque Natural Quininí, municipio de Tibacuy, Cundinamarca, Colombia. Rev. Biodivers. Neotrop. 5 (1): 7-15

Montoya, A. Briones, D. S. Nuñez, L. A. Kong, A. Ortiz, H. V. Moreno, F. A. (2019). Los hongos conocidos por la comunidad Yuhmu de Ixtenco, Tlaxcala, México. Scientia Fungorum, 49, 1-15. DOI: 10.33885/sf.2019.49.1230

Nieto-Aguilar, J. R. (2018). Guancasco: Cultura, tradición e identidad. Raíces: Revista Nicaragüense De Antropología, 2(3), 63-73. https://doi.org/10.5377/raices.v2i3.6899

Piñeros-Suárez, J. C. (2021). El interaccionismo simbólico: oportunidades de investigación en el aula de clase. Revista Internacional De Pedagogía E Innovación Educativa, 1(1), 211-228. https://doi.org/10.51660/ripie.v1i1.33 


\section{Raíces}

Ramírez, T. Montoya, A. Caballero, N. (2014). Una mirada al conocimiento tradicional sobre los hongos tóxicos en México. In: Moreno-Fuentes A. R. Garibay-Orijel (eds.) Estado del Arte de la Etnomicología mexicana: Hacia un Proyecto Etnomicológico Nacional

Rossetti, T. (2019). Dinámicas Eco protectora: Sanación y Apache en Nandayuri como sistema de buen vivir. Raíces: Revista Nicaragüense De Antropología, (5), 47-55. https://doi.org/10.5377/raices.v3i5.8802

Ruan, S. F. Ordaz, V. M. (2015). Aproximaciones a la etnomicología Maya. Revista Pueblos y Fronteras Digital,10 (20), 44-69. En: http://www.redalyc.org/articulo. oa? id $=90643038003$

Ruan, S. (2018). Recolección de hongos comestibles silvestres y estrategias para el reconocimiento de especies tóxicas entre los tsotsiles de Chamula, Chiapas, México. Scientia Fungorum, 48 (1),1-13. DOI:10.33885/sf.2018.48.1179

Servín, C. L. Alarcón, C. P. (2018). Conocimiento tradicional de los hongos silvestres comestibles en la comunidad p'urhépecha de Comachuén,Nahuatzen, Michoacán. Acta Universitaria, 28 (1), 2018. DOI: https://doi.org/10.15174/au.2018.1277

Ureta, F. (2020). La noción de la realidad humana en Zubiri. Revista Guatemalteca de Educación Superior, 3(1), 96-115. DOI: https://doi.org/10.46954/revistages.v1i1.8

Vargas, M. A. \& Zúñiga, R. M. (2021). Las políticas en Ciencia, Innovación y Tecnología y su relación con el contexto económico mexicano. Revista Internacional De Pedagogía E Innovación Educativa, 1(1), 173-188. https://doi.org/10.51660/ripie. v1i1.31 


\section{Roberto Emiliano Rico Carrillo}

Licenciado en Ciencias Ambientales de la U. Autónoma de Guerrero, cuatro años de experiencia en educación ambiental y proyectos comunitarios rurales. Áreas de actuación: educación ambiental y usos sustentables de los recursos naturales

\section{Ariana Montoya Ezquivel}

Actualmente es investigadora de la U. Autónoma de Tlaxcala, área de micología. Licenciada Biología, ENEP-Iztacala, Universidad Nacional Autónoma de México, Estado de México Maestra en Ciencias Biológicas, Facultad de Ciencias, Universidad Nacional Autónoma de México (Ciudad de México, México, 1997) Doctora en Biología, Facultad de Ciencias, Universidad Nacional Autónoma de México (Ciudad de México, México, 2005).

\section{Juan Camilo Cardona Castaño}

Administrador Ambiental y de los Recursos Naturales, U. Santo Tomas y doctorante en Ciencias Ambientales U. Autónoma de Guerrero, experiencia en educación ambiental y proyectos de gestión ambiental comunitaria

\section{Ezequiel Alberto Cruz Campuzano}

Licenciado en Biología del Instituto de Ciencias Biológicas, Universidad de Ciencias y Artes de Chiapas (UNICACH), fotógrafo de naturaleza y trabaja las líneas de ecología de hongos, tres años de experiencia trabajando con comunidades rurales, aprovechamiento agroecológico y micológico. 